\title{
Die neurologisch-neurochirurgische Frührehabilitation 2016
}

Neurol Rehabil 2016; 22(3): 183-186

(C) Hippocampus Verlag 2016

DOI 10.14624/NR150816.001

\author{
C.-W. Wallesch
}

\section{Zusammenfassung}

DRG-System und Fortschritte der Intensivmedizin haben den Bedarf an neurologischer Frührehabilitation als Weiterbehandlung nach schweren neurologischen und neurochirurgischen Erkrankungen sowie bei neurologischen Komplikationen und Komorbiditäten bei weiterhin bestehender Intensivtherapie oder -überwachungspflicht deutlich gesteigert. Die neurologische Frührehabilitation Phase B dürfte mittlerweile in Deutschland über mehr als 4.00o Behandlungsplätze verfügen. Es ist dem schnellen Ausbau dieses Subsektors geschuldet, dass noch Versorgungs- und regulatorische Lücken bestehen. Lösungsmöglichkeiten dafür werden aufgezeigt.

Schlüsselwörter: neurologische Frührehabilitation, Phase B, akutstationäre Krankenhausversorgung, G-DRG-System

BDH-Klinik Elzach

\section{Einleitung}

Die Bedeutung der neurologischen Frührehabilitation für die akutstationäre Krankenhausbehandlung als Weiterbehandler noch intensivüberwachungs- oder intensivtherapiepflichtiger Patienten ist in den letzten Jahren stetig gestiegen [7]. Das hat eine Reihe von Gründen [16, 17]:

- Im DRG-System ändert sich die Rolle der Akutkrankenhäuser in Richtung auf eine Zunahme der Notfallversorgung und spezialisierter Interventionen.

- In den Akutkrankenhäusern ist es zu einer qualitativen und quantitativen Ausweitung der intensivmedizinischen Behandlungsmöglichkeiten gekommen.

- Insbesondere in der Kardiochirurgie haben hochkomplizierte und komplikationsträchtige Eingriffe zugenommen.

- Die Zahl außerklinischer Reanimationen ist angestiegen.

- Es hat sich medizinisch wie ökonomisch als sinnvoll erwiesen, nach Abschluss spezialisierter Interventionen noch intensivüberwachungspflichtige und noch kontrolliert beatmete Patienten in die Weiterbehandlung strukturell und personell entsprechend ausgestatteter Kliniken zu verlegen.

\section{Standortbestimmung}

Die neurologische Frührehabilitation agiert an der Schnittstelle zwischen akutstationärer Intensivmedizin und weiterführender Rehabilitation. Für letztere ist die Fähigkeit zu kooperativer Mitarbeit unabdingbare Voraussetzung. Dies entspricht der Rehabilitations-Richtlinie des Gemeinsamen Bundesausschusses [2], die Rehabilitationsfähigkeit definiert: »Rehabilitationsfähig sind Versicherte, wenn sie aufgrund ihrer somatischen und psychischen Verfassung die für die Durchführung und Mitwirkung bei der Leistung zur medizinischen Rehabilitation notwendige Belastbarkeit und Motivation oder Motivierbarkeit besitzen« (S. 7).

Neben der Frührehabilitation, die in die Akutversorgung eingebunden ist, wie etwa auf der Stroke Unit (OPS 8-981), kennt der OPS als Leistungskatalog akutstationärer Operationen und Prozeduren folgende Arten der Frührehabilitation:

- Geriatrische frührehabilitative Komplexbehandlung (OPS 8-550)

- Neurologisch-neurochirurgische Frührehabilitation (OPS 8-552)

- Frührehabilitative Komplexbehandlung von Patienten mit Kopf-Hals-Tumoren (OPS 8-553)

- Fachübergreifende und andere Frührehabilitation (OPS 8-559)

Von diesen ist nur die neurologisch-neurochirurgische Frührehabilitation auf die Behandlung noch kooperationsunfähiger Patienten ausgelegt, sodass auch nicht neurologische oder neurochirurgische Erstversorger ihre noch intensivpflichtigen Patienten dorthin $\mathrm{zu}$ verlegen trachten. Dies ist in vielen Fällen gerechtfertigt und sinnvoll, da längere Intensivbehandlungen mit Beatmung sehr häufig zu behandlungsbedürftigen neurologischen Komplikationen und Komorbiditäten führen (Critical-Illness-Neuropathie und -Myopathie, septische Enzephalopathie, hypoxische Hirnschädigung, protrahiertes Delir). Eine neurologische Haupt- oder behandlungsbedürftige Nebendiagnose ist Voraussetzung dafür, dass die OPS 8-552 erlöswirksam wird. In der Klinik des Autors machen neurologische Komplikationen und Komorbiditäten fast $40 \%$ der kontrolliert beatmet aufgenommenen Patienten aus, bei nicht beatmet zur Aufnahme 


\section{Early neurological and neurosurgical rehabilitation (ENNR) in Germany 2016}

C.-W. Wallesch

\section{Abstract}

The introduction of the diagnosis related groups (DRG) system in Germany, and progress in intensive care capabilities, have led to an increasing demand for specialized departments and hospitals for early neurological rehabilitation of patients with neurological disorders, comorbidities or complications who continue to need intensive care. Presently, there are more than 4,00o beds in specialized facilities in Germany. The system is under construction, availability varies between German states and there are still regulatory deficits. We consider possible improvements.

Keywords: early neurological and neurosurcgical rehabilitation, phase B, in-patient hospital treatment, G-DRG-system

Neurol Rehabil 2016; 22(3): 183-186

(C) Hippocampus Verlag 2016

gelangenden Patienten hingegen nur 15\% [16]. Bei den letztgenannten dominieren mit $76 \%$ weiterhin die »klassischen « Frührehabilitationsdiagnosen nicht traumatische intrakranielle Blutungen (25\%), Hirninfarkte (37\%) und Schädel-Hirn-Traumen (14\%) [11]. Diese machten in der Erhebung von Pohl et al. [11] an 16 Frührehabilitationskliniken fast $64 \%$ der Hauptdiagnosen aus.

Gerade im Bereich der Frührehabilitation beatmeter Patienten wird die Funktion der neurologisch-neurochirurgischen Frührehabilitation als Weiterbehandler für alle Intensivstationen der Maximal- und Schwerpunktversorger deutlich. Neurologische Frührehabilitationseinrichtungen mit Beatmungskapazität müssen daher über ein breites Spektrum an medizinischen Fachdisziplinen (Neurologie/Neurochirurgie, Innere Medizin, Anästhesiologie und Intensivmedizin) und diagnostischen Möglichkeiten (Bildgebung, Bronchoskopie, Schluckdiagnostik) verfügen, um ihrem Behandlungsauftrag und ihrer Position im Versorgungssystem gerecht $\mathrm{zu}$ werden [10]. Bei mehr als wenige Tage passiv beatmeten Patienten kommt es zu einer relevanten Atrophie der Atemmuskulatur [9], die ein Weaning unter neurorehabilitativen und atem-/physiotherapeutischen Strategien erforderlich macht. Nach gelungenem Weaning stellt die Dekanülierung die nächste große Herausforderung dar, den Patienten vor aufwendiger Behandlungspflege nach Entlassung zu bewahren. Sie wird durch Aspiration limitiert, daher kommt der meist fiberendoskopischen Schluckdiagnostik und der logopädischen und ergotherapeutischen Schlucktherapie herausragende Bedeutung zu [12]. Derzeit wird diese fast ausschließlich in neurologischen Rehabilitationseinrichtungen vorgehalten.

Die neurologische Frührehabilitation, die aus den Intensivstationen der Akutversorger dort längerfristig behandelte Patienten übernimmt, ist mit den Problemen der modernen Intensivmedizin konfrontiert. Dabei handelt es sich neben umfangreichen Komorbiditäten um krankenhaushygienische Probleme [13]. Ein knappes Viertel der Patienten der Phase B ist mit MRSA, einem 3- oder 4-MRGN- oder VRE-Keim besiedelt [11].
Je nach Anteil beatmeter Patienten an der Versorgung sind die Case Mix Indices (CMI) neurologischer Frührehabilitationskliniken sehr hoch. Unter den zehn Kliniken mit der höchsten Fallschwere des Krankenhaus-Reports [4] finden sich ausschließlich spezialisierte Kardiochirurgien und Kliniken der neurologischen Frührehabilitation.

\section{Therapeutische Rationale}

Die neurologische Rehabilitation arbeitet im Spannungsfeld zwischen erlittenem strukturell irreparablen Schaden, transienten Funktionsstörungen (Diaschisis, den Folgen von Ödem und Diapedeseblutungen, funktioneller Erholung nach entlasteten extrazerebralen Blutungen) und der Lernfähigkeit und Trainierbarkeit auch des geschädigten ZNS, die Restitutionen und Kompensationen gestörter Funktionen ermöglichen können. Im Zeitfenster der neurologischen Frührehabilitation (Wochen bis wenige Monate nach erlittener Schädigung) bildet sich die Prognose hinsichtlich zukünftig möglicher Teilhabe aus. Fast 10\% der Patienten (ohne wegen Komplikationen in Akuthäuser verlegte) versterben in der neurologischen Frührehabilitation Phase B, davon 2/3 im Rahmen eines Palliativprotokolls [11]. Auch hier sind medizinische, therapeutische und pflegerische Kompetenzen gefragt, die der Rehabilitation bislang fremd waren.

\section{Therapeutische Pflege}

Wichtiges Eingangskriterium der Phase $\mathrm{C}$ und damit des erfolgreichen Abschlusses der Phase B ist die Fähigkeit, täglich an mindestens zwei Therapiemaßnahmen von je etwa 30 Minuten Dauer aktiv mitarbeiten zu können [1]. Hierzu bedarf es der Weckung, Aufrechterhaltung, Bindung und Richtung von Aufmerksamkeit. Bei vielen Patienten der Phase B lässt sich Aufmerksamkeit nur zeitweise aktivieren. Hier kommt der durch Pflegende durchgeführten Therapie zentrale Bedeutung zu. Die Pflege ist auf Basis 24/7 mit dem Patienten befasst. Pflegehandlungen, z.B. Waschen, Anziehen, Körperpflege, lassen sich so gestalten, dass Aufmerksamkeit geweckt und gelenkt und der Patient aktiv in die Handlung einbezogen wird. Es konnte gezeigt werden, dass der Pflegende dabei von der Rolle des Pflegenden in ein Rollenverständnis wie bei Therapeuten wechselt [5]. Die therapeutische Pflege ist seitens der Pflegewissenschaft noch unzureichend theoretisch fundiert [5], sodass die Operationalisierung der therapeutischen Pflege im Rahmen der OPS-Ziffer 8-552 über Leistungskataloge erfolgt [6]. Die Kataloge legen Inhalte und Plausibilitätszeiten für therapeutische Pflegehandlungen fest, über die die Leistungen der aktivierendtherapeutischen Pflege den überwiegenden Teil der in der OPS-Ziffer 8-552 geforderten mindestens 300 Therapieminuten im Tagesdurchschnitt ausmachen. 


\section{Bedarf}

Ein IGES-Gutachten [7] stellte fest, dass die OPS-Position 8-552 im Jahr 2010 in Deutschland in 107 Kliniken erbracht wurde. In den Krankenhausplänen der Länder wurden zwischen fünf (NRW) und $115(\mathrm{HH})$ Betten pro Million Einwohner ausgewiesen [7]. Dabei ist $\mathrm{zu}$ beachten, dass in manchen Ländern auch Phase-BKapazitäten im Reha-Sektor (§ 111 SGBV) etabliert sind. Das IGES-Gutachten schätzt auf unsicherer Datengrundlage den Bedarf für Nordrhein-Westfalen auf 57 Betten je Million Einwohner. Dieser Bedarf wurde bereits 2010 in den Ländern Bayern, Brandenburg, Hamburg, Hessen, Sachsen und Thüringen überschritten [7]. Bezogen auf die Bundesrepublik ergäbe sich auf der Basis des IGESGutachtens [7] ein Bedarf von über 4.60o Betten der neurologischen Frührehabilitation Phase B, der tatsächliche Bedarf liegt wahrscheinlich höher [16].

Der Verlegungsdruck auf Phase-B-Plätze ist (auch aus DRG-systemimmanenten Gründen - Verlegung nach Erreichen der mittleren Verweildauer) groß, insbesondere für noch kontrolliert beatmete Patienten. Bislang ist es der Krankenhausplanung noch nicht gelungen, eine regional homogene Versorgung mit Phase-B-Plätzen sicherzustellen. In einigen Regionen finden sich mehrere Phase-B-Kliniken nahe benachbart (z.B. Nordbaden), in anderen ist die Versorgung deutlich weniger dicht (z.B. Nord-Württemberg). Es gibt viele und gute Gründe, die Phase B an Zentren ausreichender Größe zu etablieren, um Effizienzgewinne (Skaleneffekte) und ein breites diagnostisches und therapeutisches Spektrum zu realisieren. Dennoch sollte Angehörigen die Möglichkeit gegeben werden, an der Frührehabilitation noch kritisch kranker Patienten teilzuhaben. Dies liegt auch im Interesse der meisten Patienten. Auch bei der Definition von teilhabeorientierten Rehabilitationszielen oder der Ermittlung des mutmaßlichen Patientenwillens ist die Kooperation und Kommunikation mit Angehörigen oder Nahestehenden sowie dem Betreuer oder Vorsorgebevollmächtigten wichtig. Phase-B-Einrichtungen sollten bei regionaler Planung daher in z. B. 90 Minuten Fahrzeit erreichbar sein.

Knapp 40\% der in Phase B behandelten Patienten erreichen die weiterführende Rehabilitation der Phase C, sind also noch erheblich pflegebedürftig, überwiegend bewusstseinsklar, können an mehreren Therapiemaßnahmen von je etwa 30 Minuten Dauer täglich aktiv mitarbeiten und weisen keine konkrete Selbst- und Fremdgefährdung und keine schweren Störungen des Sozialverhaltens auf (verkürzt nach [2]). In Einrichtungen, die neben der Phase $\mathrm{B}$ auch die Phase $\mathrm{C}$ vorhalten, ist die Quote deutlich höher. Hier erfolgt die Umwandlung nach Phase C in der Regel nach BAR-Kriterien [1] mit einem Barthel-Index meist von unter 20. Besiedelungen mit multiresistenten Keimen stellen kein Hindernis für die Umwandlung innerhalb des Hauses dar.

\section{Schnittstellen}

Der Zugang zur Frührehabilitation Phase B ist bei Kliniken, die im akutstationären Sektor etabliert sind (§ 109 SGB V), einfach. Patienten können ohne Genehmigung der Krankenkasse zuverlegt werden, wofür nach Erreichen der mittleren Verweildauer im erstbehandelnden Krankenhaus wegen der Anreize des DRG-Systems wirtschaftliche (Plateauphase der DRG), aber auch medizinische (knappe Intensivkapazitäten, therapeutische Kompetenz der Phase-B-Einrichtung) Gründe vorliegen. Bei Kliniken, die im Reha-Sektor angesiedelt sind, bedarf die Verlegung eines Antrags an den Kostenträger. Der Zugang wird derzeit in den meisten Regionen durch die Knappheit an verfügbaren Phase-B-Plätzen begrenzt, sodass manche Maximalversorger auf die Beatmungsstationen kleinerer Krankenhäuser ausweichen, bis ein Platz in der Frührehabilitation frei wird. Eine Beschränkung der Frührehabilitation auf jüngere Patienten wäre unethisch und ist prognostisch nicht gerechtfertigt [8].

Das Ende der Phase B ist zum einen durch die PhaseC-Eingangskriterien der BAR-Empfehlungen [2] definiert, von denen die Fähigkeit, an mehreren Therapien täglich aktiv mitwirken zu können, die wichtigste ist, zum anderen durch die Kriterien der akutstationären Behandlungsbedürftigkeit. Hier werden in Deutschland von Seiten des MDK die Kriterien des German Appropriateness Evaluation Protocols (G-AEP-Kriterien [3]) angewendet, deren Angemessenheit für die Neurologische Frührehabilitation fragwürdig ist. In den meisten Fällen sehen die Kriterien vor, dass eine akutstationäre Behandlung erforderlich ist, wenn ein Kriterium der Schwere der Erkrankung und ein weiteres Kriterium, z.B. eine nur im Krankenhaus durchführbare Behandlung, erfüllt sind. Die Durchführung einer Neurologischen Frührehabilitation wird dabei von den MDK-Gutachtern nicht als Erfüllung des Kriteriums der krankenhausspezifischen Behandlung gewertet. Im Falle einer Weiterführung der Behandlung in Phase $\mathrm{C}$ werden üblicherweise die Zeiträume der "sekundären Fehlbelegung « nach PhaseC-Sätzen vergütet (wenn die Klinik auch die Phase C vorhält). In letzter Zeit ergeben sich Hinweise, dass dies von einigen Kassen mit der Begründung verweigert wird, dass zu diesem Zeitpunkt kein Antrag auf eine Rehabilitation vorlag und die Rehabilitation eine Kostenzusage erfordert hätte. Bei aus Sicht des MDK verspäteter Verlegung in eine Pflegeeinrichtung besteht für die Klinik ein erhebliches Erlösausfallsrisiko. Auch Wartezeiten auf einen Pflegeheimplatz gehen zulasten der Klinik. Bei Kliniken, die im Reha-Sektor arbeiten, ist das Ausfallsrisiko geringer, da regelmäßig Verlängerungsanträge gestellt und begründet werden müssen. Einrichtungen, die nicht über Möglichkeiten der weiterführenden Rehabilitation (Phase C) verfügen, haben häufig Probleme in der Weiterversorgung ihrer Patienten, die die Eingangskriterien der Phase $\mathrm{C}$ erfüllen oder bei denen die Kriterien für 
den akutstationären Behandlungsbedarf (aus Sicht des MDK) nicht mehr erfüllt sind. Einrichtungen, die nur die Phasen C und D vorhalten, nehmen häufig Patienten mit einem Barthel-Index unter 30 oder MRE-Keimträger nicht auf [14]. Anders als bei Akutkrankenhäusern besteht für Rehabilitationseinrichtungen keine Aufnahmeverpflichtung. In Fällen, in denen die nahtlose Verlegung in Phase C nicht möglich ist, wäre Heilmittelerbringung im Pflegeheim die systemimmanente Weiterversorgung, was bei positiver Reha-Prognose unbefriedigend ist. Die Schnittstellenprobleme am Ende der Phase B werden von Schorl und Liebold ausführlich diskutiert [14]. Die unterschiedliche Handhabung des Endes der Phase B verhindert die Kalkulation von bewerteten DRGs anstelle der DRGs B43Z, A43Z und B61B.

In Bayern hat sich eine Arbeitsgruppe des MDK und des Arbeitskreises »Rehabilitation von Schlaganfallpatienten und Schädel-Hirn-Verletzten in Bayern e.V.« auf Kriterien für einen einheitlichen Endpunkt der akutstationären Behandlungsbedürftigkeit in der neurologischen Frührehabilitation in Bayern verständigt [15], der dort die G-AEP-Kriterien ersetzen soll. Die Kriterien sind in drei Kategorien unterteilt. Aus Kategorie I genügt die Erfüllung eines Kriteriums, um den akutstationären Behandlungsbedarf für mindestens eine Woche zu begründen (z.B. Abtrainieren vom Tracheostoma, Vorhandensein von ZVK, EVD, Thoraxdrainage, Spüldrainagen), aus Kategorie II müssen zwei Kriterien erfüllt sein (z.B. Barthel-Index $<35$, spezielles Wundmanagement, Isolationspflicht), Kategorie III enthält Sonderfälle (z.B. Versuch der Dekanülierung, oraler Kostaufbau bei noch bestehender Aspirationsgefahr) und seltene Indikationen, die einer individuellen Begründung bedürfen. Es ist zu hoffen, dass diese Kriterien bundesweit Anwendung finden. Auch eine Vereinheitlichung der Struktur- und Prozessqualität z.B. nach dem Beispiel der Fachplanung Baden-Württembergs [10] wäre wünschenswert, um die differenzierte Kalkulation von Frühreha-DRGs zu ermöglichen.

\section{Fazit}

Mit der neurologischen Frührehabilitation Phase B bildet sich ein neuer, vor dem Hintergrund des DRG-Systems, der Optimierung von Behandlungsketten und der nicht immer zeitnah umfassenden Erfolge der modernen Intensivmedizin notwendiger Subsektor der akutstationären Versorgung aus: die Weiterbehandlung bis zur möglichen Prognosestellung in Einrichtungen, die groß genug sind, um Komplikationen $\mathrm{zu}$ beherrschen und auf das Störungsmuster des Patienten individuell zugeschnittene Therapien anbieten zu können [16]. Aktuell hält die neurologische Frührehabilitation Phase B in Deutschland wohl etwa 5.000 Behandlungsplätze vor. Deren Dichte ist in den einzelnen Bundesländern sehr unterschiedlich [7]. Angesichts der Spezialisierung und des Behandlungsaufwands sollte die neurologi- sche Frührehabilitation als eigenständiges Kapitel in die Krankenhausplanung einbezogen werden und eine mit der Verteilung der Maximalversorger abgestimmte regionale Versorgung sichergestellt werden $[10,16]$.

\section{Literatur}

1. Bundesarbeitsgemeinschaft für Rehabilitation (BAR). Empfehlungen zur Neurologischen Rehabilitation von Patienten mit schweren und schwersten Hirnschädigungen in den Phasen B und C. Frankfurt: BAR; 1995.

2. Gemeinsamer Bundesausschuss. Rehabilitations-Richtlinie. www.g-ba.de/downloads/39-261-1977/2014-04-17_Re-RL_Anpassung-ICF_BAnz.pdf.

3. Katalog der G-AEP-Kriterien: www.mdk.de/media/pdf/17c Anlage2_g_aep_11_10_2003.pdf.

4. Klauber J, Geraedts M, Friedrich J, Wasem J (Hrsg.). Krankenhaus-Report 2015: Schwerpunkt Strukturwandel. Stuttgart: Schattauer 2015.

5. Lautenschläger S. Therapeutische Pflege in der neurologischen (Früh-)Rehabilitation: Eine Grounded-Theory-Studie. Rehabilitation 2015; 54: 273-278.

6. Lautenschläger S, Wallesch CW. Leistungskataloge der aktivierend-therapeutischen Pflege in der neurologischen Frührehabilitation. Rehabilitation 2015; 54: 30-37.

7. Loos S, Schliwen A. Kurzfassung zum Gutachten zur Abbildung der neurologisch-neurochirurgischen Frührehabilitation im Rahmen der Krankenhausplanung. Berlin: IGES 2012.

8. Knecht S, Roßmüller J, Unrath $\mathrm{M}$ et al. Old benefit as much as young patients with stroke from high-intensity neurorehabilitation: cohort analysis. J Neurol Neurosurg Psychiatry 2015; published online first on June 11, 2015. doi:10.1136/jnnp-2015-310344.

9. Mendez-Tellez PA, Needham DM. Early physical rehabilitation in the ICU and ventilator liberation. Respir Care 2012; 57: 1663-1669.

10. Ministerium für Arbeit und Sozialordnung, Familie, Frauen und Senioren Baden-Württemberg. Neurologische Frührehabilitation Phase B. Fachplanung für Baden Württemberg. Stuttgart: Sozialministerium Baden-Württemberg 2012.

11. Pohl M, Bertram M, Bucka C et al. Rehabilitationsverlauf von Patienten in der neurologisch-neurochirurgischen Frührehabilitation: Ergebnisse einer multizentrischen Erhebung im Jahr 2014 in Deutschland. Nervenarzt 2011; 82: 753-763.

12. Prosiegel M, Riecker A, Ledl C et al. Leitlinie »Neurogene Dysphagien «. Düsseldorf: AWMF 2015.

13. Rollnik JD, Samady A-M, Grüter L. Multiresistente Erreger in der neurologisch-neurochirurgischen Frührehabilitation. Rehabilitation 2014; 53: 346-350.

14. Schorl M, Liebold D. Neurologisch-neurochirurgische Frührehabilitation in Deutschland - Aktuelle Situation, Probleme und Lösungsmöglichkeiten aus medizinischer und juristischer Sicht. Akt Neurol 2012; 39: 1-11.

15. Veröffentlichungen des Bundesverbands NeuroRehabilitation BNR e.V. www.bv-neuroreha.de/Veroeffentlichungen.59370. html.

16. Wallesch CW. Die neurologische Frührehabilitation Phase B als neuer Subsektor der akutstationären Behandlung. Akt Neurol 2016; 43: 280-284.

17. Wallesch CW. Die Rehabilitation (noch) nicht rehabilitationsfähiger Patienten in der neurologischen Frührehabilitation. Fortschr Neurol Psychiatr 2015; 83: 257-258.

Interessenvermerk

Es besteht kein Interessenkonflikt.

\section{Korrespondenzadresse:}

Prof. Dr. Claus-W. Wallesch

BDH-Klinik Elzach

Am Tannwald 1-3

79215 Elzach

Claus.Wallesch@bdh-klinik-elzach.de 